\title{
Retrospective analysis of 119 cases of pediatric acute promyelocytic leukemia: Comparisons of four treatment regimes
}

\author{
EN-QIN LI ${ }^{1}$, LING XU $^{2}$, ZHI-QUAN ZHANG ${ }^{1}$, YAN XIAO $^{1}$, HAI-XIA GUO $^{3}$, \\ XUE-QUN LUO ${ }^{4}$, QUN HU ${ }^{5}$, DONG-BO LAI ${ }^{2}$, LI-MING TU ${ }^{6}$ and RUN-MING JIN ${ }^{1}$ \\ ${ }^{1}$ Department of Pediatrics, Affiliated Union Hospital, Tongji Medical College, Huazhong University \\ of Science and Technology, Wuhan, Hubei; ${ }^{2}$ Department of Pediatrics, Guangzhou Women and \\ Children's Medical Center; ${ }^{3}$ Department of Pediatrics, Second Affiliated Hospital of Sun Yat-sen University; \\ ${ }^{4}$ Department of Pediatrics, First Affiliated Hospital of Sun Yat-sen University, Guangzhou, Guangdong; \\ ${ }^{5}$ Department of Pediatrics, Affiliated Tongji Hospital, Tongji Medical College, \\ Huazhong University of Science and Technology, Wuhan, Hubei; ${ }^{6}$ Department of Pediatrics, \\ Guangdong General Hospital, Guangdong Academy of Medical Sciences, \\ Guangzhou, Guangdong, P.R. China
}

Received January 8, 2012; Accepted March 1, 2012

DOI: $10.3892 / \mathrm{etm} .2012 .546$

\begin{abstract}
Clinical trials have demonstrated that pediatric acute promyelocytic leukemia (APL) is highly curable. Small-scale studies have reported on the treatment of APL using one or two treatment regimes. Here, we report a multiple center-based study of 119 cases of pediatric APL treated with four regimes based on all-trans-retinoic acid (ATRA). We retrospectively analyzed the clinical characteristics, laboratorial test results and treatment outcome of the pediatric APL patients. Regime 1 used an in-house developed protocol, regime 2 was modified from the PETHEMA LPA99 protocol, regime 3 was modified from the European-APL93 protocol, and regime 4 used a protocol suggested by the British Committee for Standards in Haematology. The overall complete remission rates for the four regimes were $88.9,87.5,97.1$ and $87.5 \%$, respectively, which exhibited no statistical difference. However, more favorable results were observed for regimes 2 and 3 than regimes 1 and 4 , in terms of the estimated 3.5-year disease-free survivals, relapse rates, drug toxicity (including hepatotoxicity, cardiac
\end{abstract}

Correspondence to: Dr Run-Ming Jin, Department of Pediatrics, Affiliated Union Hospital, Tongji Medical College, Huazhong University of Science and Technology, Wuhan, Hubei 430022, P.R. China

E-mail: pediatrhematol002@yahoo.cn

Dr Ling Xu, Department of Pediatrics, Guangzhou Women and Children's Medical Center, 9 Jinsui Road, Guangzhou, Guangdong 510623, P.R. China

E-mail: luoxu164@yahoo.com

Key words: acute promyelocytic leukemia, myelogenous, pediatric, chemotherapy, survival arrhythmia, and differentiation syndrome) and sepsis. In conclusion, the overall outcomes were more favorable after treatment with regimes 2 and 3 than with regimes 1 and 4 , and this may have been due to the specific compositions of regimes 2 and 3 .

\section{Introduction}

Acute myeloid leukemia (AML) accounts for approximately $20 \%$ of acute leukemia cases in children (1). Acute promyelocytic leukemia (APL) is morphologically identified as AML-M3 according to the French-American-British (FAB) classification. APL represents 5-8\% of AML cases in children (2). It is characterized by accumulation of immature granulocytes called promyelocytes, which feature bilobulated nuclei and Auer rods (3). Cytogenetically, APL promyelocytes carry a balanced reciprocal translocation between chromosomes 15 and 17, t $(15 ; 17)$, which results in a fusion protein PML/ RAR $\alpha$ encoded by the promyelocytic leukemia $(P M L)$ gene fused with the gene for retinoic acid receptor $\alpha(\operatorname{RAR} \alpha)$ (4). Immunophenotypically, APL promyelocytes express CD33, CD13 and CD117 antigens, and less frequently CD34 and human leukocyte antigen DR (5). Five decades ago APL was considered the most fatal type of acute leukemia and the treatment of APL was a nightmare for physicians; event-free survival (EFS) of APL patients including children was only $35 \%$ (6).

Due to the development of various combinations of chemotherapy based on all-trans-retinoic acid (ATRA) and anthracycline, the 5-year disease-free survival (DFS) or EFS rate of pediatric APL patients has reached $75-80 \%$ based on recent studies (7-17). Thus, APL is now curable in most cases. Recently a study on childhood AML in Japan reported a 93.1\% overall survival (OS) rate and 94\% EFS rate for patients 
treated with ATRA, anthracyclines and cytarabine (at both the induction and consolidation stages) (16). Despite of the reported high survival rate, the outcome of APL treatment in many developing countries including China appears to vary dramatically, largely depending on how the therapeutic regimes are designed and delivered $(14,18)$. A study based in Taiwan reported that the overall survival and EFS of 6 APL children treated with ATRA during induction were 83 and $67 \%$, respectively (19). Zhang et al (20) reported that 65 Chinese APL children had better outcome with EFS, DFS, and overall survival achieved 77.5, 85.4 and $88.9 \%$, respectively. However, another study found that the EFS of 16 APL children treated with an in-house protocol and 14 with a modified PETHEMA LPA99 protocol were 38 and $79.6 \%$, respectively (12). Such large variations prompted us to identify which components included in the widely used regimes play a pivotal role in prognosis of the disease. Here, we conducted a retrospective, multiple center-based study on 119 cases of pediatric APL following treatment with four different chemotherapy regimes based on ATRA. We found that the overall outcomes were more favorable after treatment with regimes 2 and 3 than with regimes 1 and 4, and this added benefit may have been due to the presence of a Chinese herbal medicine formula, RealgarIndigo naturalis formula (RIF), and the absence of high-dose cytarabine (Ara-C) in regimes 2 and 3 .

\section{Materials and methods}

Eligibility of patients. Informed consents were obtained from the parents or guardians of the children (under the age of 18) diagnosed with APL who were enrolled at the Departments of Pediatrics, in the leukemia wards of six collaborative hospitals in China from September 1997 to December 2008. The diagnosis was based on the FAB classification, detection of the PML/RAR $\alpha$ fusion gene by RT-PCT or fluorescent in situ hybridization (FISH), and detection of $\mathrm{t}(15 ; 17)$ in bone marrow cells aspirated from the patients, as well as the morphology of the cells. Following the eligibility screening, 119 cases were retrospectively enrolled in this study. The patients were divided into four groups based on the therapeutic regimes received, with $36,16,35$ and 32 patients in regimes 1-4, respectively as described below.

Treatment. The therapeutic regimes consisted of multistage treatments including induction and consolidation (for all 4 regimes), maintenance (for regimes 2, 3 and 4), and reinforcement (for regime 3 only) (Fig. 1). Regime 1 used a protocol developed in-house including ATRA, daunomycin (DNR), Novantrone (NVT), and high-dose Ara-C $\left(2 \mathrm{~g} / \mathrm{m}^{2}, \mathrm{IV}\right)$. Regime 2 used a modified PETHEMA LPA99 protocol including ATRA, methotrexate (MTX), NVT, DNR, and RIF. Regime 3 used a modified European-APL93 protocol including ATRA, RIF, DNR, NVT, DA [DNR plus low-dose Ara-C (150 mg/m², IV)], NA [NVT plus low-dose Ara-C $\left.\left(150 \mathrm{mg} / \mathrm{m}^{2}, \mathrm{IV}\right)\right]$ and 6-mercaptopurine (6MP). Regime 4 used a protocol suggested by the British Committee for Standards in Haematology, including ATRA, DNR, and Ara-C [at a low-dose $\left(200 \mathrm{mg} / \mathrm{m}^{2}, \mathrm{IV}\right)$ and high dose $\left(2 \mathrm{~g} / \mathrm{m}^{2}, \mathrm{IV}\right)$ alternatively at various stages]. The details of the regimes are shown in Fig. 1.
Supportive therapy. Supportive therapy was provided to almost all of the patients, which was crucial to prevent the development of serious or even fatal complications such as coagulopathy and retinoic acid syndrome. Counter-coagulopathy therapy included transfusions of platelets, plasma, and cryoprecipitate to maintain the fibrinogen level above $1.5 \mathrm{~g} / 1$ and the platelet count above $3 \times 10^{10} / 1$ until clinical resolution of coagulopathy (21). Retinoic acid syndrome is another complication characterized by dyspnea, unexplained fever, weight gain, peripheral edema, pulmonary nodular infiltrates and pleuropericardial effusion. It develops rapidly and is potentially fatal. Upon observation of the earliest signs of retinoic acid syndrome, treatment with high-dose steroids was initiated immediately by administering dexamethasone at $0.3 \mathrm{mg} / \mathrm{kg} / \mathrm{dose}$ twice daily intravenously for at least 4 days to the children (22). In addition, hydroxyurea was administered to control leukocytosis when required.

Definitions. Complete remission (CR) was defined according to the US National Cancer Institute criteria as the presence of less than $5 \%$ of blast cells in bone marrow aspirates (23). DFS refers to the duration from the date of diagnosis until the date of the last follow-up for any event, e.g., failure to achieve remission, relapse, secondary malignancy, or death from any cause. The DFS was calculated based on time from the date of hematologic $\mathrm{CR}$ until death or hematologic relapse.

Statistical methods. The relationship of a patient's clinical characteristics to his/her treatment outcome was analyzed using the Cox Regression Proportional Hazard Model. Multivariate analysis was used. Clinical, demographic, biologic characteristics, treatment outcomes, and toxicity of the patients were compared using $\chi^{2}$ tests, Fisher exact tests, and one-way ANOVA. DFS of the four regimes was further analyzed using the Kaplan-Meier method, and the log-rank test was used for comparison between different treatment groups. Survival rates were represented as the mean percentage \pm standard error (SE). Statistical differences were considered significant at a P-value (2-sided) $<0.05$ or highly significant at a P-value $(2$-sided $)<0.01$.

\section{Results}

Clinical characteristics and laboratory test results of the patients. The 119 patients included 80 males and 39 females. Data were retrieved for a median follow-up time of 43 months (ranging from 1 to 147 months). Approximately half of the patients were from rural areas. The median age of the patients was 8.9 years, and $3.4 \%$ of the patients were under the age of 2. Paleness, hemorrhage, and organomegalies were present in $90.8,74.6$ and $35.5 \%$ of the patients, respectively. Fever was found in $63.8 \%$ of the patients, and bone pain was experienced by $22.7 \%$ of the patients.

Laboratorial test results of the 119 patients indicated that the median white blood cell (WBC) count was $8.79 \times 10^{9} / 1$ (range, $0.9 \times 10^{9} / 1$ to $191 \times 10^{9} / 1$ ), the median hemoglobin level was $71 \mathrm{~g} / \mathrm{dl}$ (range, 25-117 g/dl), and the median platelet number was $28 \times 10^{9} / 1$ (range, $4 \times 10^{9} / 1$ to $344 \times 10^{9} / 1$ ). Elevated lactic dehydrogenase $(\mathrm{LDH})(>450 \mathrm{U} / \mathrm{l})$ was present in 34 of 106 tested patients, of whom $5.7 \%$ (6/106) had LDH levels over 1,000 U/l. The immunophenotyping for CD13, CD33 and 


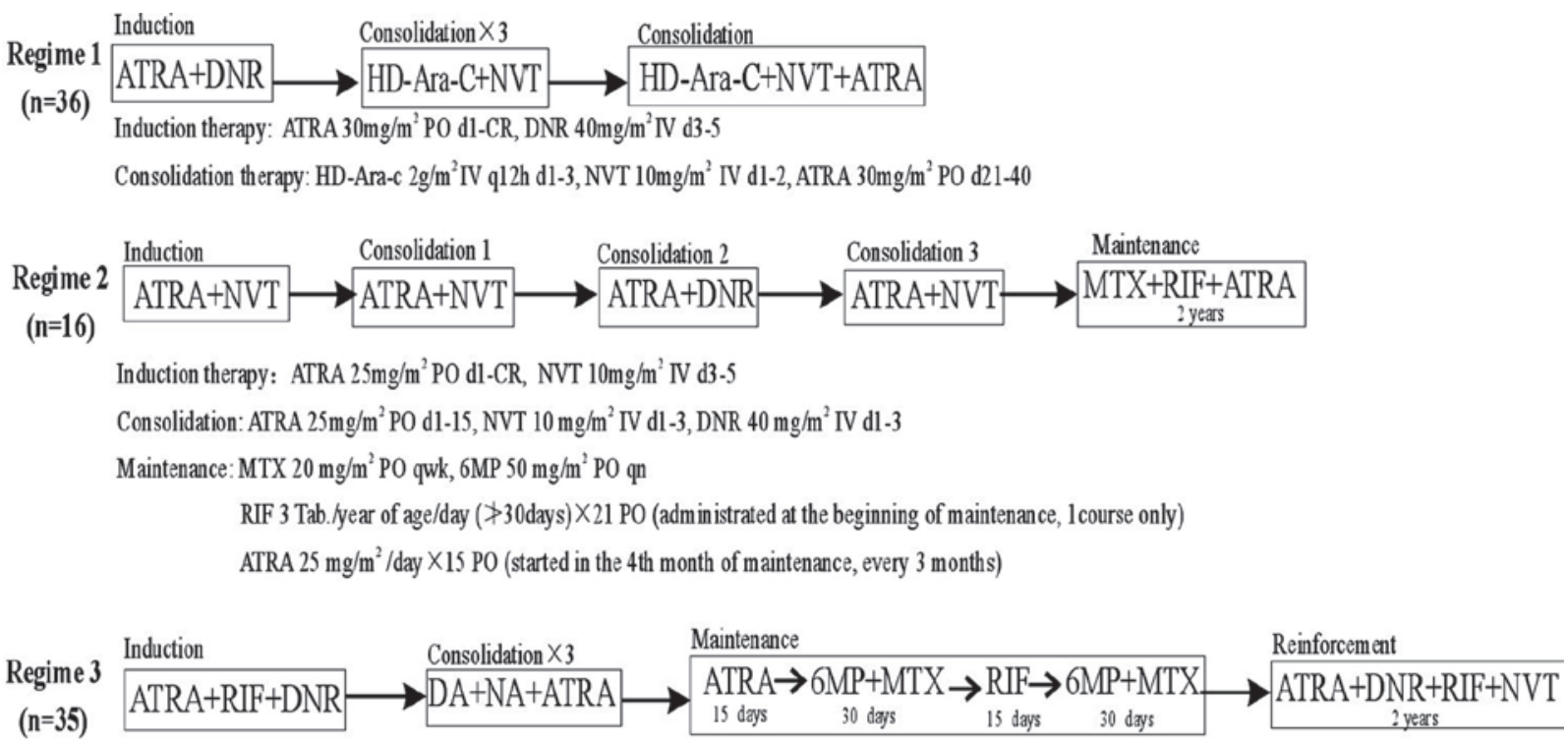

Induction: ATRA $30 \mathrm{mg} / \mathrm{m}^{2} \mathrm{PO} \mathrm{dl}-\mathrm{CR}, \mathrm{RIF} 2 \# \sim 7 \# \mathrm{PO} \mathrm{dl}-7 \rightarrow 6 \# \sim 10 \# \mathrm{PO} \mathrm{d} 21-53 \rightarrow \mathrm{CR}, \mathrm{DNR} 30 \mathrm{mg} / \mathrm{m}^{2} \mathrm{IV} \mathrm{dl} 0, \mathrm{dl} 2, \mathrm{dl} 4$

Consolidation: DA: DNR 30mg/m ${ }^{2}$ IV dl-3, Ara-c $150 \mathrm{mg} / \mathrm{m}^{2} \mathrm{IV} \mathrm{dl-7}$

NA: NVT $10 \mathrm{mg} / \mathrm{m}^{2} \mathrm{IV} \mathrm{dl}-3$, Ara-c $150 \mathrm{mg} / \mathrm{m}^{2} \mathrm{IV} \mathrm{dl}-7$

ATRA $25 \mathrm{mg} / \mathrm{m}^{2} \mathrm{P} 0 \mathrm{dl}-5$

Maintenance: ATRA $25 \mathrm{mg} / \mathrm{m}^{2} \mathrm{PO}$ dl-15, $6 \mathrm{MP} 50 \mathrm{mg} / \mathrm{m}^{2} \mathrm{PO}$ qn, MTX $20 \mathrm{mg} / \mathrm{m}^{2} \mathrm{P} 0$ qw, RFF $6 \# \sim 10 \#$ P0 $\times 15 \mathrm{~d}$

Reinforcement:ATRA $25 \mathrm{mg} / \mathrm{m}^{2} \mathrm{PO} \mathrm{dl}-15$, DNR 30 $\mathrm{mg} / \mathrm{m}^{2}$ IV dl-3, RIF 6\# 10\# PO dl-15, NVT $5 \mathrm{mg} / \mathrm{m}^{2} \mathrm{IV}$ dl-3

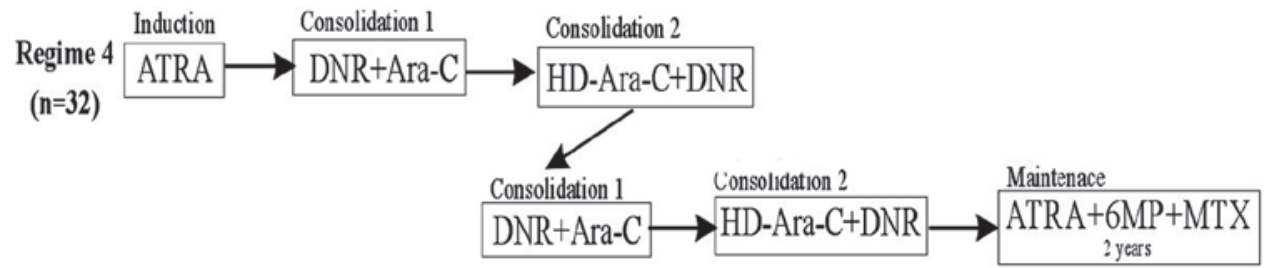

Induction: ATRA $30 \mathrm{mg} / \mathrm{m}^{2} \mathrm{P} 0 \mathrm{dl}-\mathrm{CR}$, DNR $20 \mathrm{mg} / \mathrm{m}^{\prime} \mathrm{IV} \mathrm{d} 8-10$, Ara-c $75 \mathrm{mg} / \mathrm{m}^{2} \mathrm{H}$ q12h d8-14

Consolidation 1: DNR $60 \mathrm{mg} / \mathrm{m}^{2} \mathrm{IV} \mathrm{dl-3,} \mathrm{Ara-c} 200 \mathrm{mg} / \mathrm{m}^{2} \mathrm{IV} \mathrm{dl}-7$

Consolidation 2: DNR $45 \mathrm{mg} / \mathrm{m}^{2}$ IV dl-3, HD-Ara-c $2 \mathrm{~g} / \mathrm{m}^{2} \mathrm{q} 12 \mathrm{~h}$ IV d4-7

Once a month, twice for whole therapy

Maintenance Therapy: ATRA $45 \mathrm{mg} / \mathrm{m}^{2} \mathrm{P} 0 \mathrm{dl}-15$

$6 \mathrm{MP} 90 \mathrm{mg} / \mathrm{m}^{2} \mathrm{PO}$ after ATRA

MTX $15 \mathrm{mg} / \mathrm{m}^{2} /$ wk PO after ATRA

Maintenance once every three months, 2 years for whole therapy

Figure 1. Therapeutic regimes and patient groups included in the study. Ara-C, cytarabine; ATRA, all-trans-retinoic acid; DNR, daunomycin; HD, high-dose; DA (DNR and Ara-C); MTX, methotrexate; NVT, Novantrone; 6MP, 6-mercaptopurine; NA (NVT and Ara-C); RIF, Realgar-Indigo naturalis formula; PO, per os; IV, intravenously; d, day; wk, week; q12h, every 12 hours; qwk, every week; qn, every night; Tab./\#, tablets; IH, hypodermic injection.

MPO was carried out on 91 of the 119 patients, and the rates of positive specimens were $98.3,97.2$ and $87 \%$ for the three antigens, respectively. Our data demonstrated no significant difference between the four regimes in terms of age, gender, WBC count, hemoglobin level, platelet count, and LDH level of the patients tested before the treatment (Table I).

Treatment outcome. After the remission induction, the CR rate for the four regimes was 88.9 (32/36), 87.5 (14/16), 97.1 $(34 / 35)$ and $87.5 \%(28 / 32)$, respectively, without statistical difference between the regimes. The 3.5-year DFS for the four treatment regimes was 72.2 $\pm 8.6,87.5 \pm 8.3,93.6 \pm 4.4$ and $64.6 \pm 13 \%$, respectively, with the DFS for regime 2 statistically higher than that for regime $1(\mathrm{P}<0.05)$, and DFS for regime 3 statistically higher than those for regimes $1(\mathrm{P}<0.01)$ and $4(\mathrm{P}<0.01)$ (Table II and Fig. 2). The relapse rate for the 4 regimes was $25,6.3,5.7$ and $28.1 \%$, respectively, with a statistically significant difference detected between regimes 1 and $2(\mathrm{P}<0.05)$, and between regimes 3 and $4(\mathrm{P}<0.05)$ (Table II and Fig. 2). Although all four regimes resulted in high CR rates, regimes 1 and 4 were associated with lower DFS and higher relapse rates than regimes 2 and 3 . Thus, it appears that 
Table I. Clinical characteristics and laboratory results at diagnosis of the APL patients classified into the four treatment groups.

\begin{tabular}{|c|c|c|c|c|c|}
\hline Characteristics & Regime 1 & Regime 2 & Regime 3 & Regime 4 & P-value \\
\hline Age, years, median (range) & $7.7(1.6-12.4)$ & $8.2(2.2-17.5)$ & $8.7(0.5-14.6)$ & $10(2.2-14.9)$ & 0.196 \\
\hline Male/female (n) & $26 / 10$ & $9 / 7$ & $22 / 13$ & $20 / 12$ & 0.065 \\
\hline WBC, $10^{9} / 1$, range (median) & $1.2-127.5(3)$ & $1.1-143.4(7.7)$ & $0.2-145.7(11.1)$ & $1.9-191(32.3)$ & 0.055 \\
\hline $\mathrm{Hb}, \mathrm{g} / \mathrm{l}$, range (median) & $25-116(70)$ & $38-98(76)$ & $32-117(72)$ & $34-128(71)$ & 0.500 \\
\hline Platelet, $10^{9} / 1$, range (median) & $4-344(29)$ & $7-190(30)$ & $8-164(26)$ & $9-146(40)$ & 0.984 \\
\hline LDH, U/1, range (median) & $153-1,314(245)$ & $120-1,561(357)$ & $28-1,470(283)$ & $15-1,995(314)$ & 0.607 \\
\hline
\end{tabular}

WBC, white blood cells; Hb, hemoglobin; LDH, lactate dehydrogenase.

Table II. Outcome of the patients following treatment with the four different regimes.

\begin{tabular}{|c|c|c|c|c|c|c|}
\hline \multirow[b]{2}{*}{ Treatment outcome } & \multicolumn{4}{|c|}{ Regimes } & \multicolumn{2}{|c|}{ P-value } \\
\hline & 1 & 2 & 3 & 4 & $<0.05$ & $<0.01$ \\
\hline Number of cases & 36 & 16 & 35 & 32 & & \\
\hline Complete response, $\mathrm{n}(\%)$ & $32(88.9)$ & $14(87.5)$ & $34(97.1)$ & $28(87.5)$ & & \\
\hline Relapse, n (\%) & $9(25.0)$ & 1 (6.3) & $2(5.7)$ & $9(28.1)$ & $1 / 3,3 / 4$ & \\
\hline 3.5-year DFS, \% & $72.2 \pm 8.6$ & $87.5 \pm 8.3$ & $93.6 \pm 4.4$ & $64.6 \pm 13$ & $1 / 2$ & $1 / 3,3 / 4$ \\
\hline \multicolumn{7}{|l|}{ Toxicity, n (\%) } \\
\hline Hepatotoxicity & $22(61.1)$ & $3(18.8)$ & $7(20.0)$ & $15(46.9)$ & $2 / 4,3 / 4$ & $1 / 2,1 / 3$ \\
\hline Headache & $4(11.1)$ & $2(12.5)$ & $4(11.4)$ & $5(15.6)$ & & \\
\hline Skin reaction & $7(19.4)$ & $3(18.8)$ & $8(22.9)$ & $7(21.9)$ & & \\
\hline Dryness of lips & $13(36.1)$ & $4(25.0)$ & $13(37.1)$ & $11(34.4)$ & & \\
\hline Fluid retention & $3(8.3)$ & $2(12.5)$ & $10(28.6)$ & $3(9.4)$ & $1 / 3,3 / 4$ & \\
\hline Cardiac arrhythmia & $2(5.6)$ & 0 & 0 & $6(18.8)$ & $1 / 4,2 / 4$ & $3 / 4$ \\
\hline Differentiation syndrome & 0 & 0 & 0 & $4(12.5)$ & $1 / 2,2 / 4$ & $3 / 4$ \\
\hline Sepsis & $25(69.4)$ & $3(18.8)$ & $6(17.1)$ & $20(62.5)$ & & $1 / 2,1 / 3,2 / 4,3 / 4$ \\
\hline
\end{tabular}

Values are expressed as the number of cases (percentage of total case number treated with the regime). DFS, disease-free survival.

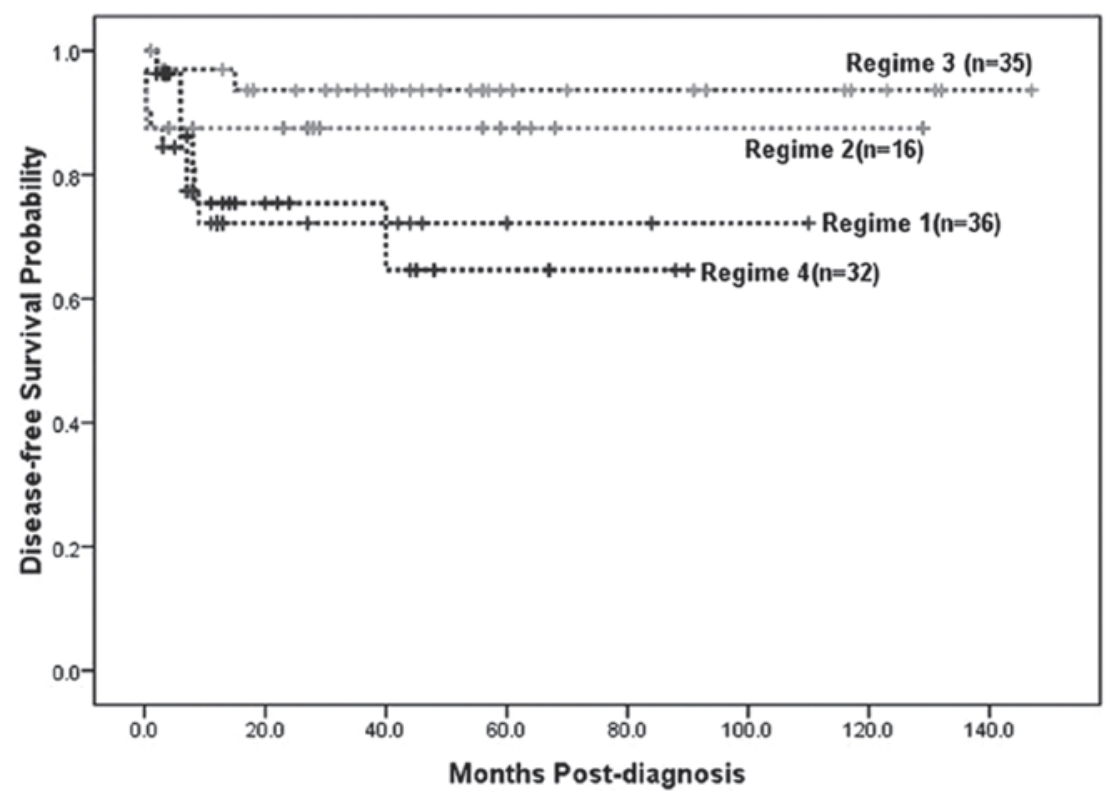

Figure 2. Disease-free survival of the APL patients treated with the four different regimes. Data were analyzed using the Kaplan-Meier survival calculation method. Vertical crossing lines indicate censors taken during the follow-up times. 
the overall outcome for regimes 2 and 3 was more favorable than that for regimes 1 and 4 .

Toxicity. The occurrence of hepatotoxicity, headache, skin reaction, dryness of lips, fluid retention, cardiac arrhythmia, differentiation syndrome, and sepsis was recorded for all the patients during all the therapeutic stages (Table II). Of note, hepatotoxicity and sepsis occurred statistically more frequently in regimes 1 and 4 than in regimes 2 and 3. Fluid retention was observed more with regime 3 than with regimes 1 and 4. Cardiac arrhythmia occurred more with regimes 1 and 4 than regimes 2 and 3. Differentiation syndrome occurred only in regime 4 . Based on the above findings, the incidence of general toxicity and sepsis was lower for treatment regimes 2 and 3 than with regimes 1 and 4 .

\section{Discussion}

In the present study, data was collected and analyzed regarding the clinical characteristics, laboratory test results and treatment outcome of 119 cases of pediatric APL treated with four different chemotherapy regimes. Similar to previous reports (7-9), the WBC counts of $47 \%$ of the patients were higher than $10 \times 10^{9} / 1$. High WBC counts combined with or without low platelet counts have been considered as indices of relapse risk $(9,17,24)$. However, we found that the WBC and platelet counts and hemoglobin and LDH levels, tested before treatment, had no significant influence on prognosis of the pediatric APL patients (data not shown). Instead, the various therapeutic regimes imposed significantly different impacts on the outcomes of the patients.

Regimes 1-4 resulted in a CR rate of $88.9,87.5,97.1$ and $87.5 \%$, respectively, a 3.5-year DFS rate of 72.2, 87.5, 93.6 and $64.6 \%$, respectively, and a relapse rate of $25,6.3,5.7$ and $28.1 \%$, respectively. Although all four regimes led to high CR rates, regimes 1 and 4 resulted in lower DFS and higher relapse than regimes 2 and 3 . Therefore, the overall outcome for regimes 2 and 3 was more favorable than regimes 1 and 4 .

It does not appear that the comparably short treatment without maintenance for regime-1 patients was accountable for their poor survival, as the regime-4 patients had maintenance treatment, yet both their CR and 3.5-year DFS rates were the lowest. Therefore, these different outcomes might have mainly resulted from the different components in the regimes. Although all four regimes included ATRA and DNR, plus other various chemotherapeutic agents, regimes 2 and 3 had two obvious features. They included the Chinese herbal medicine formula RIF, and no Ara-C (regime 2) or only low-dose $\left(150 \mathrm{mg} / \mathrm{m}^{2}\right)$ Ara-C (regime 3 ). In contrast, Ara-C was used at a high dose $\left(2 \mathrm{~g} / \mathrm{m}^{2}\right)$ during all the consolidation treatments in regime 1 , and at low doses (30 or $45 \mathrm{mg} / \mathrm{m}^{2}$ ) in two of the consolidation treatments and intermediate $\left(300 \mathrm{mg} / \mathrm{m}^{2}\right)$ and high $\left(2 \mathrm{~g} / \mathrm{m}^{2}\right)$ doses, respectively, in the other two consolidation treatments in regime 4. It appears that the use of the Chinese herbal medicine formula RIF and avoidance of the high-dose Ara-C may have reduced the treatment-related toxicity, relapse rate, and the frequency of sepsis, hence contributing to the optimal outcome of the patients treated with regimes 2 and 3 .

Traditional Chinese medicine (TCM) is a unique medical system used for thousands of years by the Chinese and other ethnic populations in China as well as many other countries. TCM doctors usually prescribe a combination of plant species or minerals to treat diseases $(25,26)$. Increasing evidence has demonstrated that TCM can be used as alternative medicine to treat diseases that have no cure using conventional medicine, or as supportive medicine to enhance therapeutic efficacy and reduce adverse effects of conventional medicine such as chemotherapy. RIF contains realgar, indigo naturalis, tetraarsenic tetrasulfide, indirubin and tanshinone IIA as its major active ingredients.

Wang et al (27) reported that RIF, when used in a murine APL model, promoted ubiquitination and degradation of the PML/RAR $\alpha$ oncoprotein by inducing expression and transportation of aquaglyceroporin-9 which degraded PML/RAR $\alpha$. It also enhanced $\mathrm{G} 1 / \mathrm{G} 0$ arrest of APL cells by regulating multiple targets of the cell cycle. Notably, recent multi-center clinical trials showed that a CR rate of $98 \%$ and a 5-year overall survival rate of $87 \%$ were achieved in adult APL patients receiving RIF, with only moderate adverse effects such as gastrointestinal discomfort and rash $(25,26,28)$. Furthermore, Luo et al (12) reported that a modified PETHEMA LPA99 protocol by including RIF had an improved overall outcome for 13 Chinese children with APL. These lines of evidence are consistent with the added beneficial effect of including RIF in regimes 2 and 3 in the present study (Table I and Fig. 2). In addition, compared to arsenic trioxide, a widely used antileukemia drug analogous to tetra-arsenic tetrasulfide, RIF is relatively inexpensive, can be taken orally and shortens the hospital stay of patients (29).

Ara-C is an anti-metabolite chemotherapeutic drug, which acts by impeding cancer cells from making and repairing DNA required for cell proliferation. Ara-C has been used to treat acute leukemia, several types of head and neck cancers, and non-Hodgkin's lymphoma. In induction or consolidation treatment for AML, high doses of either DNR or Ara-C often result in improved remission and survival rates (30-33). However, among these studies, only Weick et al (33) compared two doses of Ara-C, 1,400 and 24,000 mg/m² for induction chemotherapy, and found no difference in overall survival rate of the patients.

The dose of Ara-C used during consolidation has also been extensively explored in single-arm trials. Mayer et al (34) reported a large, randomized study of 596 patients with AML in first remission, which suggested a dose-response relationship with Ara-C. Patients who received the dose of 3,000 mg/ $\mathrm{m}^{2}$ had an improved disease-free and overall survival, especially for those who were under 60 years of age. However, an important finding of this study is that high-dose Ara-C was effective only in patients who had 'favorable', 'intermediate' or normal karyotypes upon treatment (34). As our patients all had abnormal karyotypes by the nature of the disease, high $\left(2 \mathrm{~g} / \mathrm{m}^{2}\right)$ doses of Ara-C used in the regimes may not have helped, but instead counteracted, the outcome of the young patients. One of the serious side effects of Ara-C, like many other chemotherapeutics, is increased risk of fatal infection due to reduced WBC count, which might have contributed to the short 3.5-year EFS and CR rate of the patients on regimes 1 and 4.

Overall, our present study, the largest scale study to date concerning pediatric APL in China to our knowledge, has 
revealed important information for the treatment of pediatric APL. We found that the modified PETHEMA LPA99 protocol (for the regime 2) and the modified European ALP93 protocol (regime 3), both including RIF and the absence of high-dose Ara-C, achieved a more favorable overall outcome, less chemotherapy treatment-related toxicity, and lower frequency of sepsis and relapse for APL children, than the in-house protocol (regime 1) and the protocol suggested by the British Committee for Standards in Haematology (regime 4). The inclusion of RIF and exclusion of high-dose Ara-C may have contributed to the beneficial effects of regimes 2 and 3 on the prognosis of pediatric APL cases.

\section{Acknowledgements}

We thank the Huazhong University of Science and Technology and the Sun Yat-sen University for funding this study.

\section{References}

1. Smith MA, Ries LA, Gurney JG, et al (eds): Leukemia. In: Cancer incidence and survival among children and adolescents. United States SEER Program 1975-1995. National Cancer Institute, SEER Program, Bethesda, MD, pp17-34, 1999.

2. Cantu-Rajnoldi A, Biondi A, Jankovic M, et al: Diagnosis and incidence of acute promyelocytic leukemia (FAB M3 and M3 variant) in childhood. Blood 81: 2209-2210, 1993.

3. Catovsky D, Matutes E, Buccheri V, et al: A classification of acute leukaemia for the 1990s. Ann Hematol 62: 16-21, 1991

4. de Thé $\mathrm{H}$ and Chen $\mathrm{Z}$ : Acute promyelocytic leukaemia: novel insights into the mechanisms of cure. Nat Rev Cancer 10: 775-783, 2010 .

5. Lo-Coco F and Ammatuna E: The biology of acute promyelocytic leukemia and its impact on diagnosis and treatment. Hematology Am Soc Hematol Educ Program 514: 156-161, 2006.

6. Avvisati G, Petti MC, Lo-Coco F, et al: Induction therapy with idarubicin alone significantly influences event-free survival duration in patients with newly diagnosed hypergranular acute promyelocytic leukemia: final results of the GIMEMA randomized study LAP 0389 with 7 years of minimal follow-up. Blood 100: 3141-3146, 2002.

7. Mann G, Reinhardt D, Ritter J, et al: Treatment with all-trans retinoic acid in acute promyelocytic leukemia reduces early deaths in children. Ann Hematol 80: 417-422, 2001.

8. de Botton S, Coiteux V, Chevret S, et al: Outcome of childhood acute promyelocytic leukemia with all-trans-retinoic acid and chemotherapy. J Clin Oncol 22: 1404-1412, 2004.

9. Testi AM, Biondi A, Lo Coco F, et al: GIMEMA-AIEOPAIDA protocol for the treatment of newly diagnosed acute promyelocytic leukemia (APL) in children. Blood 106: 447-453, 2005.

10. Ortega JJ, Madero L, Martin G, et al: Treatment with all-trans retinoic acid and anthracycline monochemotherapy for children with acute promyelocytic leukemia: a multicenter study by the PETHEMA Group. J Clin Oncol 23: 7632-7640, 2005.

11. Powell B, Moser B, Stock W, et al: Preliminary results from the North American acute promyelocytic leukemia (APL) study C9710 (abstract). Blood 108: 566, 2006.

12. Luo XQ, Ke ZY, Huang LB, et al: Improved outcome for Chinese children with acute promyelocytic leukemia: a comparison of two protocols. Pediatr Blood Cancer 53: 325-328, 2009.

13. Gregory J, Kim H, Alonzo T, et al: Treatment of children with acute promyelocytic leukemia: results of the first North American Intergroup trial INT0129. Pediatr Blood Cancer 53: 1005-1010, 2009.

14. Jeddi R, Ghedira H, Ben Abdennebi Y, et al: ATRA and anthracycline-based chemotherapy in the treatment of childhood acute promyelocytic leukemia (APL): a 10-year experience in Tunisia. Med Oncol 28: 1618-1623, 2010.

15. Creutzig U, Zimmermann M, Dworzak M, et al: Favourable outcome of patients with childhood acute promyelocytic leukaemia after treatment with reduced cumulative anthracycline doses. Br J Haematol 149: 399-409, 2010.
16. Imaizumi M, Tawa A, Hanada R, et al: Prospective study of a therapeutic regimen with all-trans retinoic acid and anthracyclines in combination of cytarabine in children with acute promyelocytic leukaemia: the Japanese childhood acute myeloid leukaemia cooperative study. Br J Haematol 152: 89-98, 2011.

17. Kim MH CC, Lee JW, Jang PS, et al: Outcome of childhood acute promyelocytic leukemia treated using a modified AIDA protocol. Korean J Hematol 45: 236-241, 2010.

18. Ribeiro RC and Rego E: Management of APL in developing countries: epidemiology, challenges and opportunities for international collaboration. Hematology Am Soc Hematol Educ Program 2006: 162-168, 2006.

19. Yeh TC, Liu HC, Wang LY, et al: The development of a novel protocol for the treatment of de novo childhood acute myeloid leukemia in a single institution in Taiwan. J Pediatr Hematol Oncol 29: 826-831, 2007.

20. Zhang L, Zhao H, Zhu X, et al: Retrospective analysis of 65 Chinese children with acute promyelocytic leukemia: a single center experience. Pediatr Blood Cancer 51: 210-215, 2008.

21. Avvisati G, Lo Coco F, Diverio D, et al: AIDA (all-trans retinoic acid + idarubicin) in newly diagnosed acute promyelocytic leukemia: a Gruppo Italiano Malattie Ematologiche Maligne dell'Adulto (GIMEMA) pilot study. Blood 88: 1390-1398, 1996.

22. De Botton S, Chevret S, Coiteux V, et al: Early onset of chemotherapy can reduce the incidence of ATRA syndrome in newly diagnosed acute promyelocytic leukemia (APL) with low white blood cell counts: results from APL 93 trial. Leukemia 17: 339-342, 2003.

23. Cheson BD, Cassileth PA, Head DR, et al: Report of the National Cancer Institute-sponsored workshop on definitions of diagnosis and response in acute myeloid leukemia. J Clin Oncol 8: 813-819, 1990.

24. Sanz MA, Lo Coco F, Martin G, et al: Definition of relapse risk and role of nonanthracycline drugs for consolidation in patients with acute promyelocytic leukemia: a joint study of the PETHEMA and GIMEMA cooperative groups. Blood 96: 1247-1253, 2000.

25. Huang S, Guo A, Xiang Y, et al: Clinical study on the treatment of acute promyelocytic leukemia with composite Indigo Naturalis tablets. Chin J Hematol 16: 26-28, 1995.

26. The cooperation group of phase II. Clinical trial of compound Huangdai tablet: phase II clinical trial of compound Huangdai tablet in newly diagnosed acute promyelocytic leukemia. Chin J Hematol 12: 801-804, 2006.

27. Wang L, Zhou GB, Liu P, et al: Dissection of mechanisms of Chinese medicinal formula Realgar-Indigo naturalis as an effective treatment for promyelocytic leukemia. Proc Natl Acad Sci USA 105: 4826-4831, 2008

28. Xiang Y: The influence on long-term survey of the patients with acute promyelocytic leukemia treated with compound Huangdai tablets and chemotherapy. Chin J Clin Hematol 16: 204-206, 2007.

29. Rizzari C and Biondi A: Tailoring treatment strategy for acute promyelocytic leukemia in low-income countries. Pediatr Blood Cancer 53: 303-305, 2009.

30. Preisler H, Davis RB, Kirshner J, et al: Comparison of three remission induction regimens and two postinduction strategies for the treatment of acute nonlymphocytic leukemia: a cancer and leukemia group B study. Blood 69: 1441-1449, 1987.

31. Dillman RO, Davis RB, Green MR, et al: A comparative study of two different doses of cytarabine for acute myeloid leukemia: a phase III trial of Cancer and Leukemia Group B. Blood 78: 2520-2626, 1991.

32. Schiller G, Gajewski J, Nimer S, et al: A randomized study of intermediate versus conventional-dose cytarabine as intensive induction for acute myelogenous leukaemia. Br J Haematol 81: 170-177, 1992.

33. Weick JK, Kopecky KJ, Appelbaum FR, et al: A randomized investigation of high-dose versus standard-dose cytosine arabinoside with daunorubicin in patients with previously untreated acute myeloid leukemia: a Southwest Oncology Group study. Blood 88: 2841-2851, 1996.

34. Mayer RJ, Davis RB, Schiffer CA, et al: Intensive postremission chemotherapy in adults with acute myeloid leukemia. Cancer and Leukemia Group B. N Engl J Med 331: 896-903, 1994. 